Pacific

Journal of

Mathematics

\title{
THE ABSOLUTE GALOIS GROUP OF $C(x)$
}

Dan Haran and Moshe Jarden 


\title{
THE ABSOLUTE GALOIS GROUP OF $C(x)$
}

\author{
Dan Haran AND Moshe Jarden
}

We use elementary algebraic methods to reprove a theorem which was proved by Pop using rigid analytic geometry and in a less general form by Harbater using formal algebraic patching:

Let $C$ be an algebraically closed field of cardinality $m$. Consider a subset $S$ of $\mathbb{P}^{1}(C)$ of cardinality $m$. Then the fundamental group of $\mathbb{P}^{1}(C) \backslash S$ is isomorphic to the free profinite group of rank $m$.

We also observe that if $\operatorname{char}(C) \neq 0$ and $0<\operatorname{card}(S)<m$, then $\pi_{1}\left(\mathbb{P}^{1}(C) \backslash S\right)$ is not isomorphic to a free profinite group.

\section{Introduction.}

The goal of this note is to provide an elementary algebraic proof of the following result:

Main Theorem. Let $C$ be an algebraically closed field of cardinality $m$. Let $x$ be a transcendental element over $C$. Then the absolute Galois group of $C(x)$ is the free profinite group $\hat{F}_{m}$ of rank $m$.

The Main Theorem was first proved in characteristic 0 [Dou, Thm. 2]. The essential part of the proof, for $C=\mathbb{C}$, uses algebraic topology and complex analysis, specifically, the Riemann Existence Theorem, to give a detailed description of the relative Galois group of the maximal Galois extension of $C(x)$ ramified at most at finitely many given points of $\mathbb{P}^{1}(C)$. (See the survey $[\mathbf{J a} \mathbf{1}, \S 1]$.) Unfortunately, this proof fails in positive characteristic. Worse, in this case, the structure of the relative Galois group is still unknown. Nevertheless, it is possible to prove that $G(C(x))$ is free by solving finite embedding problems over $C(x)$. Indeed, if $\operatorname{card}(C)=\aleph_{0}$, a criterion of Iwasawa reduces the proof to showing that each finite embedding problem over $C(x)$ has a solution. If $m>\aleph_{0}$, then, by Chatzidakis' criterion, it suffices to prove that each finite embedding problem over $C(x)$ has $m$ distinct solutions.

There is a standard way to construct $m$ solutions to a given embedding problem. If $\beta$ is an ordinal number of cardinality less than $m$ and if for each $\alpha<\beta$, Solution ${ }_{\alpha}$ is a solution to the embedding problem, then one constructs Solution $_{\beta}$ such that it has a new branch point. 
Harbater [Har] and Pop [Pop] have (independently) carried out this construction. Harbater uses formal patching in his construction. Pop applies methods of rigid analytic geometry. Both methods rely on heavy machineries, which have also been applied in Raynaud's proof of Abhyankar's conjecture and its generalization by both authors.

For the purpose of proving the Main Theorem, it suffices, however, to use the more elementary technique of algebraic patching which Völklein and the first author introduced in $[\mathbf{H a V}]$ and which resulted, among others, in the proof of the Main Theorem for $m=\aleph_{0}$. The work $[\mathbf{H a V}]$ was followed by [HJ1] and [HJ2]. Both works apply algebraic patching to solve embedding problems, however, ignoring ramification. The present note therefore complements $[\mathbf{H a V}],[\mathbf{H J} \mathbf{1}]$, and $[\mathbf{H J} 2]$ and fills up the gap of $[\mathbf{H J} \mathbf{1}]$ and [HJ2] by taking care of ramification. As a result we provide here an elementary algebraic proof of the Main Theorem.

It turns out that the same method allows us to prove the freeness of certain fundamental groups. Let $S$ be a subset of $C \cup\{\infty\}$ with $\operatorname{card}(S)=m$. Denote the compositum of all finite Galois extensions of $C(x)$ unramified outside $S$ by $E_{S}$. Then $\mathcal{G}\left(E_{S} / C(x)\right)$ is called the fundamental group of $\mathbb{P}^{1}(C) \backslash S$ and is usually denoted by $\pi_{1}\left(\mathbb{P}^{1}(C) \backslash S\right)$. If $S=C \cup\{\infty\}$, then $E_{S}$ is the separable closure of $E$ and $\pi_{1}\left(\mathbb{P}^{1}(C) \backslash S\right)=G(C(x))$. We prove by algebraic patching that $\pi_{1}\left(\mathbb{P}^{1}(C) \backslash S\right) \cong \hat{F}_{m}$ (Theorem 3.4). Harbater [Har] uses formal patching to prove the same result in the case where $C \backslash S$ is a finite set. Pop [Pop] uses rigid patching to prove a stronger result: $\pi_{1}(X(C) \backslash S) \cong \hat{F}_{m}$ for any irreducible projective curve $X$ over $C$ and for each subset $S$ of $X(C)$ of cardinality $m$.

Using complex analytic methods, notably the Riemann Existence Theorem, one proves in characteristic 0 that if $S$ is finite, then $\pi_{1}\left(\mathbb{P}^{1}(\mathbb{C}) \backslash S\right)$ is a free profinite group. Indeed, the result is much stronger and allows to deduce the freeness of $\pi_{1}\left(\mathbb{P}^{1}(C) \backslash S\right)$ for an arbitrary algebraically closed field $C$ of characteristic 0 and for an arbitrary subset of $C \cup\{\infty\}$. If $\operatorname{char}(C)>0$ and $S$ is finite, then by $[$ Ser $], \pi_{1}\left(\mathbb{P}^{1}(C) \backslash S\right)$ is not free. We point out here (Theorem 3.6), that if $\operatorname{card}(S)<m$, then $\pi_{1}\left(\mathbb{P}^{1}(C) \backslash S\right.$ ) is not free. So, the results of the preceding paragraph are optimal.

\section{Ramification.}

Let $K$ be a field and let $E$ be the field of rational functions of one variable over $K$, say, $E=K(x)$. Each $\alpha \in \tilde{K} \cup\{\infty\}$ defines a $K$-place $\phi: E \rightarrow$ $\tilde{K} \cup\{\infty\}$ by $\phi(x)=\alpha$. Let us denote the corresponding prime divisor of $E / K$ (the equivalence class of $\phi$ ) by $\mathfrak{p}_{x, \alpha}$. Then $\mathfrak{p}_{x, \alpha}=\mathfrak{p}_{x, \beta}$ if and only if $\alpha, \beta$ are conjugate over $K$ (letting $\infty$ to be conjugate only to itself). Thus we may identify the prime divisors of $E / K$ with the conjugacy classes of $\tilde{K} \cup\{\infty\}$. 
Let $F / E$ be a finite extension. An element $\alpha \in \tilde{K} \cup\{\infty\}$ is a branch point of $F / E$ (with respect to $x$ ) if $\mathfrak{p}_{x, \alpha}$ is ramified in $F$. Denote the set of all branch points of $F / E$ with respect to $x$ by $\operatorname{Branch}_{x}(F / E)$; this set is finite.

Remark 1.1. Every $K$-automorphism $\theta$ of $E=K(x)$ is given by $\theta(x)=$ $\frac{a x+b}{c x+d}$, where $\left(\begin{array}{ll}a & b \\ c & d\end{array}\right) \in \mathrm{Gl}_{2}(K)$. It induces

(i) a permutation $\theta^{\prime}$ of $\tilde{K} \cup\{\infty\}$ by $\theta^{\prime}(\alpha)=\frac{a \alpha+b}{c \alpha+d}$; and

(ii) a permutation $\theta^{*}$ of the set of prime divisors of $E / K$ by mapping the equivalence class of the place $\phi$ onto the equivalence class of $\phi \circ \theta$.

In particular, $\theta(x)$ is another generator of $E / K$. It is easy to check that

$$
\theta^{*}\left(\mathfrak{p}_{x, \alpha}\right)=\mathfrak{p}_{x, \theta^{\prime}(\alpha)} \quad \text { and } \quad \mathfrak{p}_{\theta(x), \theta^{\prime}(\alpha)}=\mathfrak{p}_{x, \alpha} .
$$

Furthermore, let $F / E$ be a finite extension, and extend $\theta$ to an isomorphism of fields $F \rightarrow \theta(F)$. Then $\theta(F)$ is a finite extension of $E$ and we have

$$
\begin{aligned}
\theta^{\prime}\left(\operatorname{Branch}_{x}(\theta(F) / E)\right) & =\operatorname{Branch}_{x}(F / E), \\
\theta^{\prime}\left(\left(\operatorname{Branch}_{x}(F / E)\right)\right) & =\operatorname{Branch}_{\theta(x)}(F / E) .
\end{aligned}
$$

Indeed, let $\alpha \in \tilde{K} \cup\{\infty\}$ and let $\phi^{\prime}: E \rightarrow \tilde{K} \cup\{\infty\}$ be the representative of $\mathfrak{p}_{x, \alpha}$ given by $\phi^{\prime}(x)=\alpha$. Then $\phi^{\prime} \circ \theta: E \rightarrow \tilde{K} \cup\{\infty\}$ represents $\theta^{*}\left(\mathfrak{p}_{x, \alpha}\right)=$ $\mathfrak{p}_{x, \theta^{\prime}(\alpha)}$. If $\psi^{\prime}: \theta(F) \rightarrow \tilde{K} \cup\{\infty\}$ extends $\phi^{\prime}$, then $\psi^{\prime} \circ \theta: F \rightarrow \tilde{K} \cup\{\infty\}$ extends $\phi^{\prime} \circ \theta$. Clearly $\psi^{\prime}$ ramifies in $\theta(F) / E$ if and only if $\psi^{\prime} \circ \theta$ ramifies in $F / E$. Therefore $\mathfrak{p}_{x, \alpha}$ is ramified in $\theta(F) / E$ if and only if $\mathfrak{p}_{x, \theta^{\prime}(\alpha)}$ is ramified in $F / E$. This proves the first equation of (2).

Furthermore, $\alpha \in \operatorname{Branch}_{x}(F / E)$ if and only if $\mathfrak{p}_{x, \alpha}$ is ramified in $F / E$ if and only if $\mathfrak{p}_{\theta(x), \theta^{\prime}(\alpha)}$ is ramified in $F / E$ if and only if $\theta^{\prime}(\alpha) \in \operatorname{Branch}_{\theta(x)}(F / E)$.

To simplify the notation, write $\operatorname{Branch}(F / E)$ instead of $\operatorname{Branch}_{x}(F / E)$ from now on.

For the rest of this section assume that $K$ is complete under a non-trivial ultrametric absolute value || . Extend || from $K$ to $E$ by $\left|\sum a_{n} x^{n}\right|=$ $\max _{n}\left|a_{n}\right|, a_{n} \in K$.

Let $I \neq \emptyset$ be a finite set. Let $c_{i} \in K$, for $i \in I$, such that $\left|c_{i}\right| \leq\left|c_{i}-c_{j}\right|=1$ for $i \neq j$. For each $i \in I$ put $w_{i}=\frac{1}{x-c_{i}} \in K(x)$. Let $R=K\left\{w_{i} \mid i \in I\right\}$ be the completion of the subring $K\left[w_{i} \mid i \in I\right]$ of $E$. Thus (cf. [HJ1, Lemma 3.3]) each element $f$ of $R$ has a unique presentation as a multiple power series:

$$
f=a_{0}+\sum_{i \in I} \sum_{n=1}^{\infty} a_{i n} w_{i}^{n},
$$

where $a_{0}, a_{i n} \in K$, and $\left|a_{i n}\right| \rightarrow 0$ as $n \rightarrow \infty$. Moreover, $|f|=\max _{i, n}\left\{\left|a_{0}\right|\right.$, $\left.\left|a_{i n}\right|\right\}$. Let $Q=\operatorname{Quot}(R)$ be the quotient field of $R$.

Extend the absolute value || from $K$ to $\tilde{K}$ (uniquely, since $K$ is complete). 
Lemma 1.2. Let $\mathfrak{p}_{x, \alpha}$ be a prime divisor of $E / K$ and let $v$ be be the associated discrete valuation of $E / K$.

(a) If $\left|\alpha-c_{i}\right| \geq 1$ for all $i \in I$, then $v$ extends to a valuation $\hat{v}$ of $Q$ such that the extension $(Q, \hat{v}) /(E, v)$ is immediate.

(b) Let $F / E$ be a finite Galois extension such that $F \subseteq Q$. If $\alpha \in$ $\operatorname{Branch}(F / E)$, then there is $i \in I$ such that $\left|\alpha-c_{i}\right|<1$.

Proof. (a) The map $\phi: R \rightarrow K(\alpha)$ given by

$$
a_{0}+\sum_{i \in I} \sum_{n=1}^{\infty} a_{i n} w_{i}^{n} \mapsto a_{0}+\sum_{i \in I} \sum_{n=1}^{\infty} a_{i n}\left(\frac{1}{\alpha-c_{i}}\right)^{n}
$$

is clearly an epimorphism of rings. Fix $i \in I$. By [HJ1, Prop. 3.9] and its proof, $R$ is a principal ideal domain and the ideal $\operatorname{Ker}(\phi)$ of $R$ is generated by an element $q \in K\left[w_{i}\right]$ such that $\operatorname{Ker}(\phi) \cap K\left[w_{i}\right]=q K\left[w_{i}\right]$.

Since $q$ is irreducible in $R$, the localization $R_{q R}$ is a discrete valuation ring, and hence $\phi$ uniquely extends to a place $\phi: Q \rightarrow K(\alpha) \cup\{\infty\}$. Clearly, $\phi$ extends $\mathfrak{p}_{x, \alpha}$. Thus the corresponding discrete valuation $\hat{v}$ on $Q$ extends $v$. It has the same residue field $K(\alpha)$ as $v$ has, and $q$ is a uniformizer for both $v$ and $\hat{v}$. Therefore $\hat{v} / v$ is immediate.

(b) Suppose that $\left|\alpha-c_{i}\right| \geq 1$ for each $i \in I$. By (a), $v$ extends to $Q$ such that the extension is immediate; in particular, it is unramified. But $E \subseteq F \subseteq Q$, hence $v$ is unramified in $F$.

Now assume that $I$ has at least 2 elements. For each $i \in I$ let

$$
Q_{i}=\operatorname{Quot}\left(K\left\{w_{j} \mid j \neq i\right\}\right) \quad \text { and } \quad Q_{i}^{\prime}=\operatorname{Quot}\left(K\left\{w_{i}\right\}\right) .
$$

Then

(3a) $\bigcap_{i \in I} Q_{i}=E$ and $Q_{i}^{\prime}=\bigcap_{j \neq i} Q_{j}$, for each $i \in I$ [HJ1, Prop. 3.10];

(3b) For each positive integer $n$ and for all $B \in \mathrm{GL}_{n}(Q)$ and $i \in I$ there exist $B_{1} \in \mathrm{GL}_{n}\left(Q_{i}\right)$ and $B_{2} \in \mathrm{GL}_{n}\left(Q_{i}^{\prime}\right)$ such that $B=B_{1} B_{2}[\mathbf{H J} 1$, Cor. 4.5].

Furthermore, let $G_{i} \leq G, i \in I$, be finite groups and $F_{i}, i \in I$, be fields such that

(3c) $F_{i} / E$ is a Galois extension with group $G_{i}, i \in I$;

(3d) $F_{i} \subseteq Q_{i}^{\prime}$;

(3e) $G=\left\langle G_{i} \mid i \in I\right\rangle$.

Remark 1.3. Conditions (3a)-(3e) amount to saying that $\mathcal{E}=\left(E, F_{i}, Q_{i}, Q\right.$; $\left.G_{i}, G\right)_{i \in I}$ is a patching data in the sense of $[\mathbf{H a V}$, Definition 3.3], [HJ1, Definition 1.1], and [HJ2, Definition 3.1]. In what follows we shall consider the compound $F$ of $\mathcal{E}$. As explained in [HaV, Lemma 3.6], $F$ is a certain Galois extension of $E$ contained in $Q$ with Galois group $G$. More precisely, by (3a) we have for each $i \in I$ that $Q_{i} \cap Q_{i}^{\prime}=E$, and hence the restriction map of Galois groups $\mathcal{G}\left(F_{i} Q_{i} / Q_{i}\right) \rightarrow \mathcal{G}\left(F_{i} / E\right)=G_{i}$ is an isomorphism. If 
we identify $\mathcal{G}\left(F_{i} Q_{i} / Q_{i}\right)$ with $G_{i}$ via this map, then $F$ is the largest subfield of $\bigcap_{i \in I} F_{i} Q_{i}$, on which $G$ acts so that each subgroup $G_{i}$ of $G$ acts via the restriction of automorphisms to $F[\mathbf{H a V}$, Lemma 3.6(b),(c)].

Lemma 1.4. Let $F$ be the compound of $\mathcal{E}=\left(E, F_{i}, Q_{i}, Q ; G_{i}, G\right)_{i \in I}$.

(a) Let $i \in I$. If $\alpha \in \operatorname{Branch}\left(F_{i} / E\right)$, then $\left|\alpha-c_{i}\right|<1$. In particular, the sets $\operatorname{Branch}\left(F_{i} / E\right)$, for $i \in I$, are disjoint.

(b) $\operatorname{Branch}(F / E)=\bigcup_{i \in I} \operatorname{Branch}\left(F_{i} / E\right)$.

(c) Suppose that the set I contains the symbol 1 and $G=H \ltimes G_{1}$, where $H=\left\langle G_{i} \mid i \in I \backslash\{1\}\right\rangle \triangleleft G$. Then $F^{H}=F_{1}$ and $\operatorname{Branch}\left(F^{G_{1}} / E\right)=$ $\bigcup_{\substack{i \in 1 \\ i \neq 1}} \operatorname{Branch}\left(F_{i} / E\right)$.

Proof. (a) By assumption, $F_{i} \subseteq Q_{i}^{\prime}$. By Lemma 1.2(b), with $I=\{i\}$, each $\alpha \in \operatorname{Branch}\left(F_{i} / E\right)$ satisfies $\left|\alpha-c_{i}\right|<1$.

(b) Let $\mathfrak{p}_{x, \alpha}$ be prime divisor of $E / K$ and let $v$ be the corresponding discrete valuation of $E$. Assume first that $v$ is ramified in $F_{i}$. By (a), $\left|\alpha-c_{i}\right|<1$. For each $j \neq i$ we have $\left|c_{i}-c_{j}\right|=1$, hence $\left|\alpha-c_{j}\right|=1$. By Lemma 1.2(a), $v$ extends to a valuation $v_{i}$ on $Q_{i}$ which is immediate in $Q_{i} / E$. By $[\mathbf{H a V}$, Lemma 3.6(e)], $v$ is ramified in $F$.

Conversely, assume that $v$ is ramified in $F$. We claim that there is $i \in I$ such that

$$
\left|\alpha-c_{j}\right| \geq 1 \text { for all } j \neq i .
$$

Indeed, if there is $i \in I$ such that $\left|\alpha-c_{i}\right|<1$, then $i$ satisfies $(*)$, because $\left|c_{i}-c_{j}\right|=1$ for all $j \neq i$. Otherwise each $i \in I$ satisfies $(*)$.

Fix $i \in I$ that satisfies $(*)$. By Lemma 1.2(a), $v$ extends to a valuation $v_{i}$ on $Q_{i}$ which is immediate in $Q_{i} / E$. By $[\mathbf{H a V}$, Lemma 3.6(e)], $v$ is ramified in $F_{i}$.

(c) We have $F^{H}=F_{1}$ by [HJ2, Cor. 3.4(d)] with $\Gamma=1$. It follows that $F_{1} \cap F^{G_{1}}=E$ and $F_{1} F^{G_{1}}=F$. Hence $\operatorname{Branch}(F / E)=\operatorname{Branch}\left(F_{1} / E\right) \cup$ $\operatorname{Branch}\left(F^{G_{1}} / E\right)$.

Let $\alpha \in \bigcup_{i \neq 1} \operatorname{Branch}\left(F_{i} / E\right)$. By (b), $\alpha \in \operatorname{Branch}(F / E)$; but, by (a), $\alpha \notin \operatorname{Branch}\left(F_{1} / E\right)$. Hence $\alpha \in \operatorname{Branch}\left(F^{G_{1}} / E\right)$.

Conversely, let $\alpha \in \operatorname{Branch}\left(F^{G_{1}} / E\right)$. Then $\alpha \in \operatorname{Branch}(F / E)$. By (b), there is $i \in I$ such that $\alpha \in \operatorname{Branch}\left(F_{i} / E\right)$. If $i=1$, then, as in the first paragraph of the proof of (b), the valuation $v$ corresponding to $\mathfrak{p}_{x, \alpha}$ extends to a valuation $v_{i}$ on $Q_{i}$ which is immediate in $Q_{i} / E$. But $F^{G_{1}} \subseteq$ $\left(F_{1} Q_{1}\right)^{G_{1}}=Q_{1}$, so $v_{i}$ is ramified over $v$. A contradiction. Therefore $\alpha \in$ $\bigcup_{i \neq 1} \operatorname{Branch}\left(F_{i} / E\right)$.

\section{The fundamental group of a subset of a line.}

Let $K$ be an algebraically closed field, and fix a transcendental element $x$ over $K$. The set $P$ of prime divisors of $E=K(x)$ can be identified with $K \cup\{\infty\}$. For each subset $S$ of $P$ let $E_{S}$ be the maximal Galois extension of 
$E$ unramified outside $S$, and let $G_{S}(E)=\mathcal{G}\left(E_{S} / E\right)$. In particular, $G_{P}(E)$ is the absolute Galois group $G(E)$ of $E$.

The main result of this section is that if $K$ is complete with respect to a non-trivial ultrametric absolute value, and $\operatorname{card}(K \backslash S)<\operatorname{card}(K)$, then $G_{S}(E)$ is the free profinite group of cardinality card $(K)$.

Recall [FrJ, p. 289] that a finite embedding problem for a profinite group $G$

$$
(\alpha: B \rightarrow A, \phi: G \rightarrow A)
$$

consists of an epimorphism $\alpha: B \rightarrow A$ of finite groups and a continuous epimorphism $\phi: G \rightarrow A$. The kernel of (1) is $\operatorname{Ker} \alpha$. A solution (resp. a weak solution) is a continuous epimorphism (resp. homomorphism) $\psi: G \rightarrow B$ such that $\alpha \circ \psi=\phi$.

Without loss of generality $\phi$ is the quotient map modulo $\operatorname{Ker} \phi$. Thus if $G$ is a Galois group, say $G=\mathcal{G}(\hat{E} / E)$, then $A=\mathcal{G}\left(F_{1} / E\right)$, where $F_{1}$ is a finite Galois extension of $E$ contained in $\hat{E}$, and $\phi$ is the restriction map from $\hat{E}$ to $F_{1}$. In this case we usually abbreviate (1) as

$$
\alpha: B \rightarrow \mathcal{G}\left(F_{1} / E\right) .
$$

A solution field of (2) is a Galois extension $F$ of $E$ such that $E \subseteq F_{1} \subseteq$ $F \subseteq \hat{E}$ with an isomorphism $\lambda: \mathcal{G}(F / E) \rightarrow B$ such that $\alpha \circ \lambda=\operatorname{res}_{F / F_{1}}$. By Galois theory, the solutions fields $F$ of (2) correspond to the kernels of the solutions $\psi: G(\hat{E} / E) \rightarrow B$. Notice that only finitely many solutions may have the same kernel.

We begin with a weaker assertion:

Lemma 2.1. Let $S \subseteq P$. Then $G_{S}(E)$ is projective, i.e., every finite embedding problem for $G_{S}(E)$ has a weak solution.

Proof. If $S$ is finite, this is the content of [Ser, Prop. 1] or [Ja2, Theorem 2.7]. (If $S=\emptyset$ then $G_{S}(E)=1$ by the Riemann-Hurwitz genus formula [FrJ, Prop. 2.15].)

In the general case we have to show that each finite embedding problem (2) for $G_{S}(E)$ has a weak solution. Here $F_{1} \subseteq E_{S}$.

Let $T=\operatorname{Branch}\left(F_{1} / E\right)$. Then $T \subseteq S$, and hence $F_{1} \subseteq E_{T} \subseteq E_{S}$. Factor the restriction $\phi: G_{S}(E) \rightarrow \mathcal{G}\left(F_{1} / E\right)$ into the restrictions $\operatorname{res}_{1}: G_{S}(E) \rightarrow$ $G_{T}(E)$ and $\operatorname{res}_{2}: G_{T}(E) \rightarrow \mathcal{G}\left(F_{1} / E\right)$. As $T$ is finite, by the above quoted result there is a homomorphism $\psi_{2}: G_{T}(E) \rightarrow B$ such that $\alpha \circ \psi_{2}=\operatorname{res}_{2}$. Put $\psi=\psi_{2} \circ \operatorname{res}_{1}$. Then $\alpha \circ \psi=\operatorname{res}_{F_{1}}$.

Lemma 2.2. For each integer $n>1$ there exists a cyclic extension $F / E$ of degree $n$ such that $\operatorname{Branch}(F / E)=\{1, \infty\}$. If $\operatorname{char}(K)>0$ and $n$ is a power of $\operatorname{char}(K)$, then there exists a cyclic extension $F / E$ of degree $n$ such that $\operatorname{Branch}(F / E)=\{\infty\}$. 
Proof. If $\operatorname{char}(K) \nmid n$, let $F=E(y)$, where $y^{n}=x-1$. If $n=p=\operatorname{char}(K)>$ 0 , let $F=E(y)$, where either $y^{p}-y=x$ or $y^{p}-y=\frac{x^{2}}{x-1}$. In the first case $\operatorname{Branch}(F / E)=\{\infty\}$ and in the second case $\operatorname{Branch}(F / E)=\{1, \infty\}$.

The rest of the proof reduces the general case to these two cases.

Part A. Without loss of generality $n$ is a prime power. Indeed, if $n=$ $\prod_{i=1}^{m} p_{i}^{r_{i}}$, where $p_{1}, \ldots, p_{m}$ are distinct primes, and for each $1 \leq i \leq m$ there is a cyclic extension $F_{i} / E$ of degree $p_{i}^{r_{i}}$, ramified at $\{1, \infty\}$, then the compositum $F=\prod_{i=1}^{m} F_{i}$ has the required properties.

Part B. Without loss of generality $n$ is prime. Indeed, assume that $n$ is a power of a prime $p$ and there is a cyclic extension $F_{1} / E$ of degree $p$, ramified at $\{1, \infty\}$. Let $S=\{1, \infty\}$. By Lemma 2.1, the embedding problem

$$
\left(\alpha: \mathbb{Z} / n \mathbb{Z} \rightarrow \mathbb{Z} / p \mathbb{Z}=\mathcal{G}\left(F_{1} / E\right), \quad \text { res }: G_{S}(E) \rightarrow \mathcal{G}\left(F_{1} / E\right)\right)
$$

for $G_{S}(E)$ has a weak solution, say, $\psi: G_{S}(E) \rightarrow \mathbb{Z} / n \mathbb{Z}$. But $\psi$ is surjective, since $\alpha(\psi(\mathbb{Z} / n \mathbb{Z}))=\mathbb{Z} / p \mathbb{Z}$ and $\mathbb{Z} / n \mathbb{Z}$ is the only subgroup $H$ of $\mathbb{Z} / n \mathbb{Z}$ with $\alpha(H)=\mathbb{Z} / p \mathbb{Z}$. The fixed field $F$ of $\operatorname{Ker} \psi$ has the required properties.

Lemma 2.3. Assume that $K$ is complete with respect to a non-trivial ultrametric absolute value || . Let $c \in K$ and put $w=\frac{1}{x-c}$. Let $n>1$ be an integer. Then there is $0<r<1$ such that for all $b_{1}, b_{2} \in K$ with $\left|b_{1}-c\right|,\left|b_{2}-c\right| \leq r$ there is a cyclic extension $F / E$ of degree $n$, with $\operatorname{Branch}(F / E)=\left\{b_{1}, b_{2}\right\}$ and $F \subseteq \operatorname{Quot}(K\{w\})$.

Proof. Lemma 2.2 produces a cyclic extension $F_{1} / E$ of degree $n$ with $\operatorname{Branch}\left(F_{1} / E\right)=\{1, \infty\}$. Since $F_{1} / E$ is unramified at 0 , we have $F_{1} \subseteq$ $K((x))$. By [ HaV, Lemma 4.2(b)] there is $r>0$ with the following property: If $a \in K^{\times}$and $|a| \leq r$, then the $K$-automorphism of $E$ given by $x \mapsto a x$ extends to an embedding $\mu_{a}: F_{1} \rightarrow$ Quot $(K\{x\})$. Without loss of generality $r<1$. Let $b_{1}, b_{2} \in K$ such that $\left|b_{1}-c\right|,\left|b_{2}-c\right| \leq r$. Put $a=b_{2}-b_{1}$ and denote $F_{2}=\mu_{a}\left(F_{1}\right)$. By Remark 1.1,

$$
\operatorname{Branch}\left(F_{2} / E\right)=\left(\mu_{a}^{\prime}\right)^{-1}\left(\operatorname{Branch}\left(F_{1} / E\right)\right)=\frac{1}{a}\{1, \infty\}=\left\{\frac{1}{b_{2}-b_{1}}, \infty\right\} .
$$

Let $\theta$ be the $K$-automorphism of $E$ given by $\theta(x)=w$. Extend $\theta$ to an isomorphism of fields $\theta: F_{2} \rightarrow F_{3}$. Then $F_{3} \subseteq \operatorname{Quot}(K\{w\})$. We have

$$
\begin{aligned}
\operatorname{Branch}\left(F_{3} / E\right) & =\left(\theta^{\prime}\right)^{-1}\left(\operatorname{Branch}\left(F_{2} / E\right)\right) \\
& =\left(\theta^{\prime}\right)^{-1}\left\{\frac{1}{b_{2}-b_{1}}, \infty\right\}=\left\{c+b_{2}-b_{1}, c\right\} .
\end{aligned}
$$


Let $d=c-b_{1}$. Then $|d| \leq r \leq 1$. Let $\lambda$ be the automorphism of $K[[w]]$ that maps $f=\sum_{n=0}^{\infty} a_{n} w^{n}$ onto

$$
\begin{aligned}
\lambda(f)=\sum_{n=0}^{\infty} a_{n}(w+d)^{n} & =\sum_{n=0}^{\infty} a_{n} \sum_{k=0}^{n}\left(\begin{array}{l}
n \\
k
\end{array}\right) d^{n-k} w^{k} \\
& =\sum_{k=0}^{\infty}\left(\sum_{n=k}^{\infty}\left(\begin{array}{l}
n \\
k
\end{array}\right) a_{n} d^{n-k}\right) w^{k} .
\end{aligned}
$$

Then

$$
\left|\sum_{n=k}^{\infty}\left(\begin{array}{l}
n \\
k
\end{array}\right) a_{n} d^{n-k}\right| \leq \max _{n \geq k}\left|a_{n}\right|
$$

and hence $\lambda(K\{w\}) \subseteq K\{w\}$. Therefore we can extend $\lambda$ to an automorphism of $\operatorname{Quot}(K\{w\})$. The restriction of $\lambda$ to $E$ is the map $w \mapsto w+d$. Let $F=\lambda\left(F_{3}\right)$. Then $F \subseteq \operatorname{Quot}(K\{w\})$ and

$\operatorname{Branch}(F / E)=\left(\lambda^{\prime}\right)^{-1}\left(\operatorname{Branch}\left(F_{3} / E\right)\right)=\left\{c+b_{2}-b_{1}-d, c-d\right\}=\left\{b_{2}, b_{1}\right\}$.

To prove that a projective group is free, we need the following criterion, essentially due to Iwasawa [FrJ, Cor. 24.2] and Chatzidakis [FrJ, Lemma 24.14 and Prop. 24.18].

Lemma 2.4. Let $m$ be an infinite cardinal number and let $G$ be a projective group of rank $\leq m$. Put

$$
m^{\prime}= \begin{cases}1 & \text { if } m=\aleph_{0}, \\ m & \text { if } m>\aleph_{0},\end{cases}
$$

and assume that each finite split embedding problem for $G$ with a non-trivial kernel has $m^{\prime}$ distinct solutions. Then $G \cong \hat{F}_{m}$.

Proof. The existence of $m$ solutions of (1) for $A=1$ and $B=\mathbb{Z} / 2 \mathbb{Z}$ implies that $G$ is of rank $m$.

By [FrJ, Cor. 24.2] in the first case and by [FrJ, Lemma 24.14 and Prop. 24.18] or [Ja1, Lemma 2.1] in the second case, it suffices to prove that each (i.e., not necessarily split) finite embedding problem (1) for $G$ with $\operatorname{Ker} \alpha \neq 1$ has $m^{\prime}$ distinct solutions. As $G$ is projective, there exists a homomorphism $\psi: G \rightarrow B$ such that $\alpha \circ \psi=\phi$. Then $\hat{A}=G / \operatorname{Ker} \psi$ is a finite group and there exist homomorphisms $\hat{\phi}: \hat{A} \rightarrow A$ and $\hat{\psi}: \hat{A} \rightarrow B$ such that $\hat{\phi} \circ \pi=\phi, \hat{\psi} \circ \pi=\psi$, and $\alpha \circ \hat{\psi}=\hat{\phi}$, where $\pi: G \rightarrow \hat{A}$ is the quotient map. Let $\hat{B}=B \times_{A} \hat{A}$ and let $\hat{\alpha}: \hat{B} \rightarrow \hat{A}$ and $\beta: \hat{B} \rightarrow B$ be the projections from $\hat{B}$. Then there exists $\theta: \hat{A} \rightarrow \hat{B}$ such that $\hat{\alpha} \circ \theta=\mathrm{id}_{\hat{A}}$ and $\beta \circ \theta=\hat{\psi}$ $[$ FrJ, Lemma 20.6]. So, $(\pi: G \rightarrow \hat{A}, \hat{\alpha}: \hat{B} \rightarrow \hat{A})$ is a finite split embedding problem for $G$ and $\operatorname{Ker} \hat{\alpha} \cong \operatorname{Ker} \alpha \neq 1$. 
By assumption, there exist $m^{\prime}$ distinct epimorphisms $\psi_{i}: G \rightarrow \hat{B}$ such that $\hat{\alpha} \circ \psi_{i}=\pi, i \in I$. If $i, i^{\prime} \in I$ and $\beta \circ \psi_{i}=\beta \circ \psi_{i^{\prime}}$, then $\psi_{i}=\psi_{i^{\prime}}$ [FrJ, Lemma 20.6]. Conclude that $\beta \circ \psi_{i}, i \in I$, are $m^{\prime}$ distinct solutions of embedding problem (1).

A disk in $K \cup\{\infty\}$ is a set of the form

$$
D=\theta(\{a \in K|| a \mid \leq r\})
$$

where $r>0$ and $\theta$ is a Möbius transformation over $K$. Thus each set of the form $D=\left\{a \in K|| a-c \mid \leq r^{\prime}\right\}$ or $D=\left\{a \in K|| a \mid \geq r^{\prime}\right\} \cup\{\infty\}$, where $r^{\prime}>0$ and $c \in K$, is a disk. (In fact, each disk is of this form; but we shall not use this fact.) Note that the cardinality of a disk is the same as the cardinality of $K$.

Lemma 2.5. Assume that $K$ is complete with respect to a non-trivial ultrametric absolute value. Let $F_{1} / E$ be a finite Galois extension with group $G_{1}$. Let

$$
\alpha: G=H \rtimes G_{1} \rightarrow G_{1}=\mathcal{G}\left(F_{1} / E\right)
$$

be a finite split embedding problem for $G(E)$. Suppose that $H=\operatorname{Ker} \alpha$ is generated by a finite family $\left\{G_{i}\right\}_{i \in J}$ of non-trivial cyclic subgroups. Then there exists a family of pairwise disjoint disks $\left\{D_{i}\right\}_{i \in J}$ in $K \cup\{\infty\}$ such that for every $B \subset \bigcup_{i \in J} D_{i}$ with $\operatorname{card}\left(B \cap D_{i}\right)=2$, for each $i \in J$, there exists a solution field $F$ to (3) with $\operatorname{Branch}\left(F^{G_{1}} / E\right)=B$.

Proof. For the sake of compatibility with [HJ2] assume that $J$ does not contain the symbol 1 and put $I=J \cup\{1\}$. Then $G=\left\langle G_{i} \mid i \in I\right\rangle$. For each $i \in I$ let $c_{i} \in K, w_{i}$, etc., be as in Section 1 (see Remark 1.3). In particular, $Q_{i}=\operatorname{Quot}\left(K\left\{w_{j} \mid j \neq i\right\}\right)$ and $Q_{i}^{\prime}=\operatorname{Quot}\left(K\left\{w_{i}\right\}\right)$.

Claim. We may assume that $F_{1} \subseteq Q_{1}^{\prime}$. Indeed, as $K$ is algebraically closed, every prime divisor of $F_{1} / K$ is of degree 1 . In particular, $F_{1} / K$ has an unramified prime divisor of degree 1. By [HaV, Lemma 4.2] there is a $K$-automorphism of $E$ that extends to an embedding $\theta: F_{1} \rightarrow Q_{1}^{\prime}$. Let $F_{1}^{\prime}=\theta\left(F_{1}\right)$ and extend $\theta$ to an automorphism of $\tilde{E}$. Then $\theta$ defines isomorphisms $\theta_{*}: \mathcal{G}\left(F_{1} / E\right) \rightarrow \mathcal{G}\left(F_{1}^{\prime} / E\right)$ and $\theta_{*}: G(E) \rightarrow G(E)$ such that the following diagram commutes

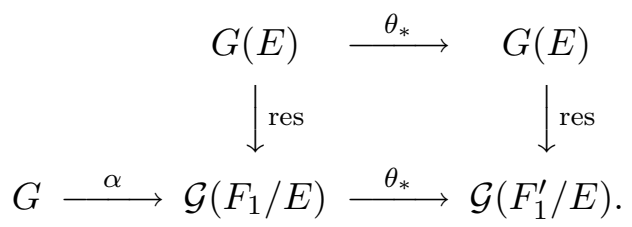

Suppose that there is a family of disks $\left\{D_{i}^{\prime}\right\}_{i \in J}$ such that for every $B^{\prime} \subset$ $\bigcup_{i \in J} D_{i}^{\prime}$ with $\operatorname{card}\left(B^{\prime} \cap D_{i}^{\prime}\right)=2$, for each $i \in J$, there exists a solution field 
$F^{\prime}$ to the embedding problem

$$
\left(\theta_{*} \circ \alpha: G \rightarrow \mathcal{G}\left(F_{1}^{\prime} / E\right), \text { res }: G(E) \rightarrow \mathcal{G}\left(F_{1}^{\prime} / E\right)\right)
$$

with $\operatorname{Branch}\left(F^{\prime G_{1}} / E\right)=B^{\prime}$. Then the disks $D_{i}=\theta^{\prime}\left(D_{i}\right)$, for $i \in J$, have the required property.

Indeed, if $B \subset \bigcup_{i \in J} D_{i}$ and $\operatorname{card}\left(B \cap D_{i}\right)=2$, for each $i \in J$, put $B^{\prime}=\left(\theta^{\prime}\right)^{-1}(B)$, and let $F^{\prime}$ be as above. Clearly, $F=\theta^{-1}\left(F^{\prime}\right)$ solves (3). By Remark 1.1, $\operatorname{Branch}\left(F^{G_{1}} / E\right)=B$.

Thus, replacing $F_{1}$ by $F_{1}^{\prime}$ we may assume that $F_{1} \subseteq Q_{1}^{\prime}$.

By Lemma 2.3 there is $0<r<1$ such that the (necessarily disjoint) disks $D_{i}=\left\{a \in K|| a-c_{i} \mid \leq r\right\}$, for $i \in J$, have the following property. For every $B \subset \bigcup_{i \in J} D_{i}$ with $\operatorname{card}\left(B \cap D_{i}\right)=2$, for each $i \in J$, there exist Galois extensions $F_{i} / E$ with the cyclic Galois group $G_{i}$ and $\operatorname{Branch}\left(F_{i} / E\right)=B \cap D_{i}$ and $F_{i} \subseteq \operatorname{Quot}\left(K\left\{w_{i}\right\}\right)$, for each $i \in J$.

By Remark $1.3, \mathcal{E}=\left(E, F_{i}, Q_{i}, Q ; G_{i}, G\right)_{i \in I}$ is a patching data. Its compound $F$ is, by [HJ2, Cor. 3.4(d)] with $\Gamma=1$, a Galois extension of $E$ that solves (3). By Lemma 1.4(c),

$$
\operatorname{Branch}\left(F^{G_{1}} / E\right)=\bigcup_{i \in J} \operatorname{Branch}\left(F_{i} / E\right)=\bigcup_{i \in J} B \cap D_{i}=B .
$$

\section{Descent.}

We wish to apply Lemma 2.5 to a sufficiently large complete extension of a given algebraically closed field.

Thus we consider the following situation. Let $C_{1} \subseteq C_{2}$ be two algebraically closed fields and let $x$ be transcendental over $C_{2}$. Denote $E_{1}=$ $C_{1}(x)$ and $E_{2}=C_{2}(x)$. Let

$$
\rho: G=H \ltimes G_{1} \rightarrow G_{1}=\mathcal{G}\left(F_{1} / E_{1}\right)
$$

be a finite split embedding problem for $G\left(E_{1}\right)$ with a non-trivial kernel. Let $F_{2}=F_{1} E_{2}$. Then the restriction $\mathcal{G}\left(F_{2} / E_{2}\right) \rightarrow \mathcal{G}\left(F_{1} / E_{1}\right)$ is an isomorphism. Identify $\mathcal{G}\left(F_{2} / E_{2}\right)$ with $G_{1}=\mathcal{G}\left(F_{1} / E_{1}\right)$ via this map. Then (1) induces a finite split embedding problem

$$
\rho: G=H \ltimes G_{1} \rightarrow G_{1}=\mathcal{G}\left(F_{2} / E_{2}\right)
$$

for $G\left(E_{2}\right)$ with a nontrivial kernel.

Before dealing with embedding problems let us notice a simple fact:

Remark 3.1. Let $A$ be an infinite subset of a field $K$. Then every nonempty Zariski $K$-open subset of $\mathbb{A}^{n}$ meets $A^{n}$. Indeed, the only polynomial in $n$ variables over $K$ that vanishes on $A^{n}$ is 0 . 
Lemma 3.2. Let $A$ be an infinite subset of $C_{1}$. Assume that (2) has a solution field $L_{2}$ such that $\infty \notin \operatorname{Branch}\left(L_{2}^{G_{1}} / E_{2}\right)$ and the elements of $\operatorname{Branch}\left(L_{2}^{G_{1}} / E_{2}\right)$ are algebraically independent over $C_{1}$. Then (1) has a solution field $L_{1}$ with $\operatorname{Branch}\left(L_{1}^{G_{1}} / E_{1}\right) \subseteq A$.

Proof. There is an irreducible monic polynomial $h \in C_{2}[x, Z]$ such that $L_{2}=$ $E_{2}(z)$, where $h(x, z)=0$. Furthermore, there are irreducible polynomials $f_{1}, \ldots, f_{r} \in C_{2}[x, Z]$ such that a root $z_{i}$ of $f_{i}$ is a primitive element of $L_{2}^{G_{1}} / E_{2}$ (and hence also of $L_{2} / F_{2}$ ), and

$$
\operatorname{Branch}\left(L_{2}^{G_{1}} / E_{2}\right)=\bigcap_{j=1}^{r} \operatorname{Discr}\left(f_{j}\right)
$$

[Has, p. 64].

There is an integer $l$ and a $l$-tuple $\mathbf{u}=\left(u_{1}, \ldots, u_{l}\right)$ of elements of $C_{2}$ such that $h, f_{1}, \ldots, f_{r} \in C_{1}[\mathbf{u}][x, Z]$. Without loss of generality $\operatorname{Branch}\left(L_{2}^{G_{1}} / E_{2}\right)$ $\subseteq\left\{u_{1}, \ldots, u_{l}\right\}$, say, $\operatorname{Branch}\left(L_{2}^{G_{1}} / E_{2}\right)=\left\{u_{1}, \ldots, u_{k}\right\}$, where $k \leq l$.

Now, u generates a variety $U=\operatorname{Spec}\left(C_{1}[\mathbf{u}]\right)$ over $C_{1}$. For each $\mathbf{u}^{\prime} \in$ $U\left(C_{1}\right)$ the $C_{1}$-specialization $\mathbf{u} \rightarrow \mathbf{u}^{\prime}$ extends to a $C_{1}(x)$-homomorphism ': $C_{1}(x)[\mathbf{u}] \rightarrow C_{1}(x)$, and from there to an $F_{1}$-homomorphism ' $: F_{1}[\mathbf{u}] \rightarrow F_{1}$. Extend it to an $F_{1}$-place from $L_{2}$ into the algebraic closure of $F_{1}$. Let $B=\left\{u_{1}^{\prime}, \ldots, u_{k}^{\prime}\right\} \subseteq C_{1}$ be the image of $\operatorname{Branch}\left(L_{2}^{G_{1}} / E_{2}\right)=\left\{u_{1}, \ldots, u_{k}\right\}$.

The variety $U$ has a nonempty Zariski open subset $U^{\prime}$ such that if $\mathbf{u}^{\prime} \in U^{\prime}$, then, in the above notation,

(4a) $h, f_{1}^{\prime}, \ldots, f_{r}^{\prime} \in C_{1}[x, Z]$ are irreducible over $C_{1}(x)$ [FrJ, Prop. 8.8];

(4b) $L_{1}=E_{1}\left(z^{\prime}\right)$ is Galois over $E_{1}$ and $\mathcal{G}\left(L_{1} / E_{1}\right) \cong \mathcal{G}\left(L_{2} / E_{2}\right)=G[\mathbf{F r J}$, Lemma 5.5];

(4c) the respective roots $z_{1}^{\prime}, \ldots, z_{r}^{\prime}$ of $f_{1}^{\prime}, \ldots, f_{r}^{\prime}$ are primitive elements for $L_{1}^{G_{1}} / E_{1}$.

Thus $L_{1}$ solves (1). From (3), $B=\bigcap_{j=1}^{r} \operatorname{Discr}\left(f_{j}^{\prime}\right)$. In particular, since $L_{1}^{G_{1}} / E_{1}$ is unramified at each point outside $\operatorname{Discr}\left(f_{1}^{\prime}\right)$,

(4d) $\operatorname{Branch}\left(L_{1}^{G_{1}} / E_{1}\right) \subseteq B$.

By assumption, $u_{1}, \ldots, u_{k}$ are algebraically independent over $C_{1}$. Thus the projection on the first $k$ coordinates pr: $U \rightarrow \mathbb{A}^{k}$ is a dominant map, and hence $\operatorname{pr}\left(U^{\prime}\right)$ contains a Zariski open subset of $\mathbb{A}^{k}$ [Lan, Prop. 4 on p. 88]. By Remark 3.1 we may choose $\mathbf{u}^{\prime}$ so that $B=\left\{u_{1}^{\prime}, \ldots, u_{k}^{\prime}\right\} \subseteq A$. Thus $\operatorname{Branch}\left(L_{1}^{G_{1}} / E_{1}\right) \subseteq A$.

To achieve the algebraic independence in Lemma 3.2 we use: 
Lemma 3.3. Let $C_{1} \subseteq C_{2}$ be two algebraically closed fields such that $\operatorname{card}\left(C_{1}\right)<\operatorname{card}\left(C_{2}\right)$. Let $\left\{D_{j}\right\}_{j \in J}$ be a finite collection of pairwise disjoint subsets of $C_{2}$ of cardinality $\operatorname{card}\left(C_{2}\right)$. Then there exists a set $B \subseteq \bigcup_{j \in J} D_{j}$ such that $\operatorname{card}\left(B \cap D_{j}\right)=2$ for each $j \in J$ and the elements of $B$ are algebraically independent over $C_{1}$.

Proof. Write $J$ as $\{1, \ldots, k\}$, and suppose, by induction, that we have already found $b_{j}, b_{j}^{\prime} \in D_{j}$, for $j=1, \ldots, k-1$, such that $b_{1}, b_{1}^{\prime}, \ldots, b_{k-1}, b_{k-1}^{\prime}$ are algebraically independent over $C_{1}$. The cardinality of the algebraic closure $\tilde{C}_{1}$ of $C_{1}\left(b_{1}, b_{1}^{\prime}, \ldots, b_{k-1}, b_{k-1}^{\prime}\right)$ in $C_{2}$ is $\operatorname{card}\left(C_{1}\right)<\operatorname{card}\left(C_{2}\right)=$ $\operatorname{card}\left(D_{k}\right)$, so there exist $b_{k}, b_{k}^{\prime} \in D_{k}$ algebraically independent over $\tilde{C}_{1}$. Thus $b_{1}, b_{1}^{\prime}, \ldots, b_{k}, b_{k}^{\prime}$ are algebraically independent over $C_{1}$.

The preceding lemmas yield the main result:

Theorem 3.4. Let $C$ be an algebraically closed field of cardinality $m$ and let $E=C(x)$ be the field of rational functions over $C$. Let $S \subseteq C \cup\{\infty\}$ of cardinality $m$. Then $G_{S}(E)$ is isomorphic to the free profinite group of rank $m$.

Proof. Put $C_{1}=C$ and $E_{1}=E$. By Lemma 2.1, $G_{S}(E)$ is projective. Therefore, by Lemma 2.4, it suffices to show that every finite split embedding problem (1) for $G_{S}(E)$ has $m^{\prime}$ solution fields, where $m^{\prime}=1$ if $m=\aleph_{0}$, and $m^{\prime}=m$ otherwise.

Let $\beta<m$ be an ordinal number. Suppose, by transfinite induction, that $\left\{L_{\alpha}\right\}_{\alpha<\beta}$ is a family of distinct solution fields of (1). For each $\alpha$, the set $\operatorname{Branch}\left(L_{\alpha} / E\right)$ is finite. Hence, $\mathrm{A}=S \backslash \bigcup_{\alpha<\beta} \operatorname{Branch}\left(L_{\alpha} / E\right)$ is infinite.

Choose an algebraically closed field $K=C_{2}$ which contains $C$, complete with respect to a non-trivial ultrametric absolute value, such that $\operatorname{card}(C)<$ $\operatorname{card}(K)$. For instance, choose a field $C^{\prime}$ that contains $C$ such that $\operatorname{card}(C)<$ $\operatorname{card}\left(C^{\prime}\right)$, and let $K$ be the completion of the algebraic closure of $C^{\prime}((t))$. Consider the induced embedding problem (2).

By Lemma 2.5 there exists a family of disks $\left\{D_{j}\right\}_{j \in J}$ in $K \cup\{\infty\}$ such that for every $B \subset \bigcup_{j \in J} D_{j}$ with $\operatorname{card}\left(B \cap D_{j}\right)=2$, for each $j \in J$, there exists a solution field $L_{2}$ to $(2)$ with $\operatorname{Branch}\left(L_{2}^{G_{1}} / K(x)\right)=B$. Choose such a set $B$. By Lemma 3.3, with $D_{j} \backslash\{\infty\}$ instead of $D_{j}$, we may assume that the elements of $B$ are algebraically independent over $C$. Therefore by Lemma 3.2, (1) has a solution field $F$ such that $\operatorname{Branch}\left(F^{G_{1}} / E\right) \subseteq A$.

Since $F=F_{1} F^{G_{1}}$, we have

$$
\operatorname{Branch}(F / E)=\operatorname{Branch}\left(F_{1} / E\right) \cup \operatorname{Branch}\left(F^{G_{1}} / E\right) .
$$

Furthermore, $\operatorname{Branch}\left(F_{1} / E\right), \operatorname{Branch}\left(F^{G_{1}} / E\right) \subseteq S$. Thus $\operatorname{Branch}(F / E) \subseteq$ $S$. Also, let $\alpha<\beta$. Then $\operatorname{Branch}\left(F^{G_{1}} / E\right) \cap \operatorname{Branch}\left(L_{\alpha} / E\right)=\emptyset$. $\operatorname{But} \operatorname{Branch}\left(F^{G_{1}} / E\right) \neq \emptyset$ by the Riemann-Hurwitz genus formula $[\mathbf{F r J}$, 
Prop. 2.15] and $\operatorname{Branch}\left(F^{G_{1}} / E\right) \subseteq \operatorname{Branch}(F / E)$. Therefore $\operatorname{Branch}(F / E)$ $\neq \operatorname{Branch}\left(L_{\alpha} / E\right)$, whence $F \neq L_{\alpha}$.

Corollary 3.5. Let $C$ be an algebraically closed field of cardinality $m$. Let $E$ be a field of algebraic functions in one variable over $C$. Then $G(E)$ is isomorphic to the free profinite group of rank $m$.

Proof. If $E$ is the field of rational functions, apply Theorem 3.4 with $S=P$. In the general case $E$ is a finite separable extension of $C(x)$. Therefore $G(E)$ is an open subgroup of $G(C(x))$. The assertion follows from $[\mathbf{F r J}$, Prop. 15.27].

If $\operatorname{char}(C)=0$ and $S$ is an arbitrary subset of $C \cup\{\infty\}$, then, using the Riemann Existence Theorem and a result of Douady for the case when $S$ is finite, one can prove that $G_{S}(E)$ is a free profinite group (as in [Ja1, §1.8]). If, however, $\operatorname{char}(C)>0$ and $\operatorname{card}(S)<\operatorname{card}(C)$, then this is no longer true. In fact, $G_{S}(E)$ is even not free:

Theorem 3.6. Let $C$ be an algebraically closed field of positive characteristic and of cardinality $m$. Let $E$ be a finite extension of $C(x)$ and let $S$ be a non-empty subset of prime divisors of $E / C$ of cardinality less than $m$. Denote the maximal Galois extension of $E$ unramified outside $S$ by $E_{S}$. Then $\mathcal{G}\left(E_{S} / E\right)$ is not a free profinite group.

Proof. Assume that $\mathcal{G}\left(E_{S} / E\right)$ is isomorphic to the free profinite group of rank $k$. For each prime number $p$ denote the maximal pro- $p$ extension of $E$ in $E_{S}$ by $E_{S}^{(p)}$. Then $\mathcal{G}\left(E_{S}^{(p)} / E\right)$ is isomorphic to the free pro- $p$ group of rank $k$. Denote the family of Galois extensions of degree $p$ in $E_{S}^{(p)}$ by $\mathcal{D}_{S}(p)$. Let $d_{S}(p)$ be the cardinality of $\mathcal{D}_{S}(p)$. Then $d_{S}(p)$ is the cardinality of the family of open normal subgroups of $\mathcal{G}\left(E_{S}^{(p)} / E\right)$ of index $p$. Hence, $d_{S}(p)$ is finite if $k$ is finite [FrJ, Lemma 15.1] and $d_{S}(p)=k$ if $k$ is infinite [FrJ, Supplement 15.2]. But this contradicts the conjunction of the following two claims.

Claim A. If $p \neq \operatorname{char}(C)$, then $d_{S}(p)<m$.

Indeed, if $S$ is a finite set, then $d_{S}(p)$ is finite [Ja2, Prop. 3.2]. In the general case, let $\mathcal{A}$ be the collection of all finite non-empty subsets of $S$. Its cardinality is, like that of $S$, less than $m$. Then $E_{S}^{(p)}$ is the compositum of all fields $E_{A}^{(p)}$, with $A \in \mathcal{A}$. Hence, $d_{S}(p) \leq \sum_{A \in \mathcal{A}} d_{A}(p)<m$.

Claim B. If $p=\operatorname{char}(C)$, then $d_{S}(p)=m$. 
Indeed, the case where $m=\aleph_{0}$ is covered by [Ja2, Prop. 3.3]. So, assume $m>\aleph_{0}$. Since $E / C(x)$ is a finite extension, it suffices to construct $m$ linearly disjoint cyclic extensions of $C(x)$ of degree $p$ unramified outside $\left.S\right|_{C(x)}$. We may therefore assume without loss that $E=C(x)$. Also, apply a Möbius transformation on $E$, if necessary, to assume that $\infty \in S$.

For each ordinal number $\alpha<m$ choose $a_{\alpha} \in C$ such that the transfinite sequence $\left(a_{\alpha} \mid \alpha<m\right)$ is linearly independent over $\mathbb{F}_{p}$. Each of the fields $E\left(z_{\alpha}\right)$ with $z_{\alpha}^{p}-z_{\alpha}=a_{\alpha} x$ is a cyclic extension of $E$ of degree $p$. Moreover, $\operatorname{Branch}_{x}\left(E\left(z_{\alpha}\right) / E\right)=\{\infty\}$. Finally, the field extensions $E\left(z_{\alpha}\right), \alpha<m$, of $E$ are linearly disjoint.

Indeed, by the theory of Artin-Schreier, it suffices to prove that the set $\left\{a_{\alpha} x \mid \alpha<m\right\}$ is linearly independent over $\mathbb{F}_{p}$ modulo $\wp(E)$, where $\wp(y)=$ $y^{p}-y$. Suppose that there exist relatively prime polynomials $f$ and $g$ in $C[x]$ such that $\sum_{\alpha<m} u_{\alpha} a_{\alpha} x=\frac{f(x)^{p}}{g(x)^{p}}-\frac{f(x)}{g(x)}$, with elements $u_{\alpha}$ in $\mathbb{F}_{p}$ which are zero for all but finitely many $\alpha$. Then each irreducible factor of $g(x)$ is a pole of the right hand side but not of the left hand side. So, we may assume that $g(x)=1$ and that $f(x)=\sum_{j=0}^{n} c_{j} x^{j}$ with $c_{j} \in C$ and $c_{n} \neq 0$. It follows that $\sum_{\alpha<m} u_{\alpha} a_{\alpha} x=\sum_{j=0}^{n} c_{j}^{p} x^{j p}-\sum_{j=0}^{n} c_{j} x^{j}$. Comparison of the coefficients of $x^{j n}$ proves that $n=0$ and $\sum_{\alpha<m} u_{\alpha} a_{\alpha}=0$. Hence, by assumption, $u_{\alpha}=0$ for each $\alpha$.

\section{References}

[Dou] A. Douady, Détermination d'un group de Galois, C.R.A.S., 258 (1964), 5305-5308.

[FrJ] M.D. Fried and M. Jarden, Field Arithmetic, Ergebnisse der Mathematik, 11(3), Springer, Heidelberg, 1986.

[HJ1] D. Haran and M. Jarden, Regular split embedding problems over complete valued fields, Forum Mathematicum, 10 (1998), 329-351.

[HJ2] _ Regular split embedding problems over function fields of one variable over ample fields, J. of Algebra, 208 (1998), 147-164.

$[\mathrm{HaV}]$ D. Haran and H. Völklein, Galois groups over complete valued fields, Israel J. of Math., 93 (1996), 9-27.

[Har] D. Harbater, Fundamental groups and embedding problems in characteristic $p$, Contemp. Math., 186 (1995), 353-369.

[Has] H. Hasse, Number Theory, Grundlehren der Math. Wissenschaften, 229, Springer, Berlin, 1980.

[Ja1] M. Jarden, On free profinite groups of uncountable rank, Contemp. Math., 186 (1995), 371-383.

[Ja2] - The projectivity of the fundamental group of the affine line, Turkish Journal of Mathematics, to appear.

[Lan] S. Lang, Introduction to Algebraic Geometry, Interscience Publishers, New York, 1958. 
[Pop] F. Pop, Étale Galois covers of affine smooth curves, Inven. Math., 120 (1995), 555-578.

[Ser] J.-P. Serre, Construction de revêtements étales de la droite affine en caracteristique p, Comptes Rendus de Académie des Sciences Paris, 311 (1990), 341-346.

Received April 28, 1999 and revised November 19, 1999. This research was supported by the Minkowski Center for Geometry at Tel Aviv University.

School of Mathematics

Tel Aviv University

Ramat Aviv, Tel Aviv 69978

ISRAEL

E-mail address: haran@math.tau.ac.il

School of Mathematics

Tel Aviv University

Ramat Aviv, Tel Aviv 69978

ISRAEL

E-mail address: jarden@math.tau.ac.il 